Supporting Information for

\title{
Core-shell and Zigzag Nanostructures from a Thin Film Silicon-containing Conformationally Asymmetric Triblock Terpolymer
}

Ling-Ying Shi, ${ }^{* a, b}$ Fen Liao, ${ }^{\mathrm{a}}$ Li-Chen Cheng, ${ }^{\mathrm{b}}$ Sangho Lee, ${ }^{\mathrm{b}}$ Rong Ran, ${ }^{\mathrm{a}}$ Zhihao Shen, ${ }^{\mathrm{c}}$ Caroline A. $\operatorname{Ross}^{*, b}$

${ }^{\mathrm{a} C o l l e g e}$ of Polymer Science and Engineering, State Key Laboratory of Polymer Materials Engineering, Sichuan University, Chengdu 610065, China

${ }^{\mathrm{b}}$ Department of Materials Science and Engineering, Massachusetts Institute of Technology, 77 Massachusetts Avenue, Cambridge, Massachusetts 02139, United States

cBeijing National Laboratory for Molecular Sciences, Key Laboratory of Polymer Chemistry and Physics of Ministry of Education, College of Chemistry and Molecular Engineering, Peking University, Beijing 100871, China

\section{Contents}

Synthesis of PDMS- $b$-PS- $b$-PMPCS triblock terpolymer through sequential ATRP

Characterization of the chemical structure and morphology of the bulk sample

Preparation and characterization of BCP films and etched patterns

Estimation of the volume fraction in the swollen film

Table S1. Molecular Weights, Volume Fractions and Tg Values of Triblock Terpolymer

Table S2. Solubility Parameters of Polymers and Solvents Studied 
Scheme S1 Synthetic route of the PDMS- $b$-PS- $b$-PMPCS triblock terpolymer.

Figure S1. ${ }^{1} \mathrm{H}-\mathrm{NMR}$ spectra of the PDMS- $b$-PS- $b$-PMPCS triblock terpolymer. $\quad$ S9

Figure S2. GPC curves of the PDMS- $b$-PS- $b$-PMPCS triblock terpolymer. S9

Figure S3. WAXS profiles of the PDMS-b-PS-b-PMPCS triblock terpolymer bulk films S10 after solvent annealing and thermal annealing at indicated conditions.

Figure S4. Schematics of DSM triblock terpolymer thin film self-assembly and subsequent plasma selective etching process.

Figure S5. Plots of total partial pressure of acetone and heptane $\left(P_{\text {ace }+ \text { hep }}\right)$ and mole fraction of the acetone in vapor $\left(f_{\text {ace, },}\right)$ as function of the mole fraction of the acetone in liquid $\left(f_{\text {ace, } \mathrm{L}}\right)$ in the vapor-liquid equilibrium acetone/heptane mixture system at different temperatures.

Figure S6. Swelling ratio profiles during solvent annealing under different solvent mixture.

Figure S7. Swelling ratios of PDMS and PS homopolymers and DSM triblock terpolymer under solvent vapor annealing with different mixed solvent ratios.

Figure S8. Representative low magnification SEM images revealing long range ordered morphologies in DSM thin films.

Figure S9. SEM images of oxidized PDMS patterns from the DSM thin films with initial thickness of $35 \mathrm{~nm}$ and $20 \mathrm{~nm}$ after annealed with acetone/heptane ratio $\sim 5: 1$.

Figure S10. SEM images of oxidized PDMS patterns after annealing with the acetone:heptane ratio $\sim 4.5: 1$ to $2.5: 1$.

Figure S11. SEM images of cylindrical oxidized PDMS patterns with bends and arrow morphology in the DSM thin film with initial thickness of $30 \mathrm{~nm}$ after annealing in vapor from acetone:heptane mixtures of 7:1.

Figure S12. SEM images of templated self-assembly of DSM terpolymer in $100 \mathrm{~nm}$ deep S16 topographic confined trenches.

\section{Synthesis of PDMS-b-PS-b-PMPCS triblock terpolymer through sequential ATRP.}

All chemicals and reagents were commercially available. Styrene and tetrahydrofuran were used after distillation. Chlorobenzene was purified by washing with concentrated sulfuric acid to remove residual thiophene, followed by washing with a $5 \%$ sodium carbonate solution and with water, and then dried with anhydrous calcium chloride before it was finally distilled. $\mathrm{CuBr}$ was purified by washing with 
acetic acid, followed by washing with methanol, and then dried for use. The macroinitiator 2-bromoisobutyrate-terminated PDMS and the monomer \{2,5-bis[(4-butoxyphenyl)oxycarbonyl] styrene $\}$ (MPCS) were synthesized as previously reported. ${ }^{1}$ All other chemicals and reagents were used as received.

The synthesis procedure of the triblock terpolymer is illustrated in scheme S1. The PDMS- $b$-PS block copolymer was synthesized first. PDMS-Br (0.20 g, $44.4 \mu \mathrm{mol})$, styrene (0.69 g, $6.6 \mathrm{mmol}), \mathrm{CuBr}$ (6.37 mg, $44.4 \mu \mathrm{mol})$, and PMDETA $(7.70 \mathrm{mg}, 44.4 \mu \mathrm{mol})$ were charged into a polymerization tube. After stirring and degassing by three freeze-pump-thaw cycles, the tube was sealed under vacuum. The tube was immersed into a $90{ }^{\circ} \mathrm{C}$ oil bath for $3 \mathrm{~h}$ for the polymerization and was then quenched in ice water. The solution was passed through a neutral alumina column to remove copper salt. Finally, the diblock copolymer was precipitated in methanol and dried in vacuum overnight.

The PDMS- $b$-PS block copolymer $(0.20 \quad \mathrm{~g}, 17.4 \mu \mathrm{mol})$, the monomer \{2,5-bis[(4-butoxyphenyl)oxycarbonyl]styrene $\}$ (MPCS, $0.91 \mathrm{~g}, 2.26 \mathrm{mmol}), \mathrm{CuBr}(2.51 \mathrm{mg}, 17.4$ $\mu \mathrm{mol})$, and PMDETA $(3.00 \mathrm{mg}, 17.4 \mu \mathrm{mol})$ were charged into a polymerization tube for polymerization and the procedure from polymerization to the dried product was similar to the preparation of PDMS- $b$-PS. Finally, the product was purified by reflux of $n$-hexane in a Soxhlet extractor for $72 \mathrm{~h}$ to remove the unreacted PDMS- $b$-PS block copolymer. The large difference in the solubility parameters between PMPCS and PDMS and between PMPCS and PS also enables the triblock copolymer to be purified by removing PDMS and PDMS- $b$-PS impurities.

\section{Characterization of the chemical structure and morphology of the bulk sample}

The chemical structure was characterized by ${ }^{1} \mathrm{H}$ NMR (Figure S1). The molecular weights of the 
PDMS- $b$-PS- $b$-PMPCS triblock terpolymer were determined from the combination of GPC, ${ }^{1} \mathrm{H}$ NMR, and the absolute molecular weight of the macroinitiator. ${ }^{1}$ Gel permeation chromatography (GPC) was conducted on a HLC-8380 instrument (Dong Chao Corporation, Japan) with pure THF as eluent (1.0 $\mathrm{mL} / \mathrm{min}$ ). The calibration curve was obtained with linear polystyrenes as standards. ${ }^{1} \mathrm{H}$ NMR spectra were obtained with a Bruker $400 \mathrm{MHz}$ spectrometer. The small-angle X-ray scattering (SAXS) and experiments were carried out on a Bruker Nanostar SAXS instrument using $\mathrm{Cu} \mathrm{K \alpha}$ radiation at a wavelength of $0.154 \mathrm{~nm}$. The working voltage and current were $40 \mathrm{kV}$ and $0.65 \mathrm{~mA}$, respectively. One-dimensional wide-angle X-ray scattering (1D WAXS) experiments were performed on a SAXSess instrument (Anton Paar) with the working voltage and current of $40 \mathrm{kV}$ and $40 \mathrm{~mA}$, respectively. The scattering vector $q$ is defined as $q=4 \pi / \lambda \sin \theta$, where the scattering angle is $2 \theta$, and the $d$-spacing $(d)$ is given by $2 \pi / q$. TEM bright field images were obtained with a Tecnai G2 F20 transmission electron microscope (SEI, USA) instrument using an accelerating voltage of $120 \mathrm{kV}$.

\section{Preparation and characterization of $\mathrm{BCP}$ films and etched patterns}

The flat substrates consisted of bare silicon wafers with a native oxide layer. Patterned silicon substrates with $100 \mathrm{~nm}$ deep trenches of different trench widths and shapes were provided by Taiwan Semiconductor Manufacturing Company (TSMC). The substrates were first functionalized by a polystyrene (PS) brush layer by spin-coating a PS-OH film ( $\sim 35 \mathrm{~nm}$ thickness) from solution $\left(M_{\mathrm{n}}=11.0\right.$ $\mathrm{kg} / \mathrm{mol}$, polydispersity index $(\mathrm{PDI})=1.1$, Polymer Source, $\mathrm{Inc})$, then baking at $170{ }^{\circ} \mathrm{C}$ under vacuum (20 Torr) for $20 \mathrm{~h}$. Upon annealing, the dehydration reaction between hydroxyl groups of the polymer chain and silanol groups of the silicon oxide led to PS chains covalently grafted on the silicon wafer. ${ }^{2,3}$ After baking, the substrates were thoroughly rinsed in toluene 5 times to remove excess polymer chains. 
The thickness of the grafted PS brush layer was measured to be $3 \mathrm{~nm}$ by optical ellipsometry.

Figure S3 schematically illustrates the process flows for fabricating self-assembled patterns in thin films of DSM triblock terpolymer on a PS brush functionalized substrate. Solutions of the DSM terpolymer were made in toluene with concentration $1.5 \sim 3.0 \mathrm{wt} \%$. Films with thickness ranging from $20 \mathrm{~nm}$ to $70 \mathrm{~nm}$ were obtained by spin-coating on substrates at various spin speeds. The initial film thickness and the thickness during solvent annealing were determined from a reflectometry system (FilMetrics F20-UV) by measuring the reflectance spectra of the BCP thin film within a wavelength range of $300-1000 \mathrm{~nm}$.

Solvent vapor annealing of the DSM thin film was carried out in a solvent reservoir annealing system consisting of a closed chamber of volume $80 \mathrm{~cm}^{3}$ with a loosely fitted lid that allowed the vapor to leak out slowly at room temperature, $23 \pm 2{ }^{\circ} \mathrm{C}$, with humidity $78 \%{ }^{4}$ The film sample was placed in the chamber supported above $5 \mathrm{~cm}^{3}$ of liquid solvent, consisting of a range of volumetric mixtures of acetone and heptane (10:0, 9:1, 7:1, 5:1, 4.5:1, 3.5:3:1, 2.5:1). According to the mass density $(\rho)$ and molecular weight $(M)$ of acetone $\left(\rho_{\text {ace }}=0.79 \mathrm{~g} / \mathrm{cm}^{3}, M_{\text {ace }}=58.08 \mathrm{~g} / \mathrm{mol}\right)$ and heptane $\left(\rho_{\text {hep }}=0.68 \mathrm{~g} / \mathrm{cm}^{3}\right.$, $\left.M_{\text {hep }}=100.21 \mathrm{~g} / \mathrm{mol}\right)$, the mole ratio between the acetone and heptane was calculated as $(20: 0,18: 1$, $14: 1,10: 1,9: 1,7: 1,6: 1,5: 1)$ corresponding to volumetric mixtures ratio of $(10: 0,9: 1,7: 1,5: 1,4.5: 1$, 3.5: $3: 1,2.5: 1)$. Based on reports of the liquid mixture of acetone/heptane ${ }^{5,6}$ similar to our solvent reservoir annealing system, the total vapor pressure or partial pressure of the solvents vapor $\left(P_{\text {ace }}+\right.$ hep $)$ and the mole fraction of acetone in vapor $\left(f_{\text {ace, }}\right)$ above the acetone/heptane liquid mixture as a function of mole fraction of acetone in the liquid $\left(f_{\text {ace, },}\right)$ at different temperatures was calculated and shown in Figure S4. The acetone/heptane is not an ideal solution and the vapor pressure quickly increases with the 
mole fraction of acetone in the liquid because the vapor pressure $(P)$ of pure acetone is much higher than that of heptane at the same temperature $\left(P_{\text {ace }}\right.$ at $273.15,298.15$, and $323.15 \mathrm{~K}$ are $9.3,30.6$ and $80.2 \mathrm{kPa}$; $P_{\text {hep }}$ at $273.15,298.15$, and $323.15 \mathrm{~K}$ are $1.5,6.0$, and $20.1 \mathrm{kPa}$, respectively. The relationship between vapor pressure and temperature is $\log \mathrm{P}=\mathrm{A}-(\mathrm{B} /(\mathrm{T}+\mathrm{C})),{ }^{5}$ with $\mathrm{A}, \mathrm{B}, \mathrm{C}$ constants. However, the partial pressures of the vapor approach equilibrium values when the $f_{\text {ace, } \mathrm{L}}$ was higher than 0.5 at $273.15 \mathrm{~K}$ and when the $f_{\text {ace, } \mathrm{L}}$ was larger than 0.70 at $313.15 \mathrm{~K}$. The solvent annealing of our experiment was performed at around $298 \mathrm{~K}$, and the $f_{\text {ace, } \mathrm{L}}$ was higher than $0.83\left(f_{\text {ace }, \mathrm{L}}=0.83\right.$ corresponds to a volume ratio of acetone/heptane 2.5:1). ${ }^{5}$ Therefore, the partial pressure of all solvent annealing processes used here is estimated to be approaching the partial pressure of acetone at $298.15 \mathrm{~K}, \sim 30.6 \mathrm{kPa}$. Although the vapor pressure did not change greatly for the all solvent annealing conditions, the mole fraction of acetone in the vapor phase continuously increased as the mole fraction of acetone in the liquid phase increased (Figure 4b), which influenced the selective swelling of the block copolymers.

The film thickness during the solvent annealing process was monitored by continuous FilMetrics F20-UV measurements. ${ }^{4}$ The swelling ratios profiles are shown in Figure S4 and S5. The solvent-annealing for times of $3 \mathrm{~h}$ to $20 \mathrm{~h}$ under an approximately steady state vapor atmosphere, the film was deswelled by quickly sliding open the lid of the chamber. The annealed BCP thin films were then subjected to a two-step reactive ion etching (Plasma-Therm 790) consisting of a $50 \mathrm{~W} \mathrm{CF}_{4}$ plasma at 15 mTorr for 3 seconds to remove the PDMS wetting layer at the film/air interface, ${ }^{3}$ and a $90 \mathrm{~W} \mathrm{O}_{2}$ plasma at 6 mTorr for 5 seconds to selectively etch the PMPCS matrix and PS shells, leaving immobilized oxidized PDMS patterns on the substrates.

The morphologies of the etched BCP thin films were characterized using a Zeiss Merlin high 
resolution scanning electron microscopy (SEM) at $2 \mathrm{kV}$, and atomic force microscopy (AFM) experiments in both height and phase-contrast modes using a Veeco Metrology Nanoscope V scanned probe microscope controller with a Dimension 3100 AFM in tapping mode.

\section{Estimation of the volume fraction in the swollen film}

The volume faction of the blocks in the swollen DSM film could be estimated from the swelling ratio values of the terpolymer, and PDMS and PS homopolymers (Figure S5). The volume fraction of the PMPCS $\left(f_{\mathrm{M}}^{\mathrm{s}}\right)$ in the swollen state is calculated using the following equation:

$$
\begin{aligned}
& f_{\mathrm{M}}{ }^{\mathrm{s}}=V_{\mathrm{M}}^{\mathrm{s}} / V_{\mathrm{DSM}}{ }^{\mathrm{s}}=\left(V_{\mathrm{DSM}^{\mathrm{s}}}-V_{\mathrm{D}}^{\mathrm{s}}-V_{\mathrm{S}}^{\mathrm{s}}\right) / V_{\mathrm{DSM}}^{\mathrm{s}} \\
& =\left(r_{\mathrm{DSM}} V_{0}-r_{\mathrm{D}} f_{\mathrm{D}} V_{0}-r_{\mathrm{S}} f_{\mathrm{S}} V_{0}\right) / r_{\mathrm{DSM}} V_{0}=\left(r_{\mathrm{DSM}}-r_{\mathrm{D}} f_{\mathrm{D}}-r_{\mathrm{S}} f_{\mathrm{S}}\right) / r_{\mathrm{DSM}}
\end{aligned}
$$

where $V_{i}^{s}$ is the volume of the $i$ polymer or the triblock terpolymer in the swollen state, $r_{i}$ is the swelling ratio of the $i$ polymer or the triblock terpolymer, and the $f_{i}$ is the initial volume fraction of the $i$ block. Therefore, the calculated $f_{\mathrm{M}}^{\mathrm{s}}$ values in the annealing condition are given as follows:

$$
\begin{aligned}
& \mathrm{A}: \mathrm{H}=5: 0, f_{\mathrm{M}}^{\mathrm{s}}=(2.15-1.6 \times 0.11-2.3 \times 0.16) / 2.15=0.746 \\
& \mathrm{~A}: \mathrm{H}=5: 1, f_{\mathrm{M}}{ }^{\mathrm{s}}=(2.05-2.05 \times 0.11-2.2 \times 0.16) / 2.05=0.718 \\
& \mathrm{~A}: \mathrm{H}=4: 1, f_{\mathrm{M}}{ }^{\mathrm{s}}=(1.90-2.10 \times 0.11-2.10 \times 0.16) / 1.90=0.701 \\
& \mathrm{~A}: \mathrm{H}=3.5: 1, f_{\mathrm{M}}^{\mathrm{s}}=(1.85-2.25 \times 0.11-2.10 \times 0.16) / 1.85=0.684
\end{aligned}
$$

Table S1. Molecular Weights and Volume Fractions of PDMS, PS and PMPCS Blocks,

Polydispersity Indexes, and $T_{\mathrm{g}}$ Values of the PS and PMPCS Blocks in the Triblock Terpolymer

\begin{tabular}{cccccccccc}
\hline Sample & $\begin{array}{c}M_{\mathrm{n}, \mathrm{D}}{ }^{a} \\
(\mathrm{~g} / \mathrm{mol})\end{array}$ & $\begin{array}{c}M_{\mathrm{n}, \mathrm{S}}{ }^{a} \\
(\mathrm{~g} / \mathrm{mol})\end{array}$ & $\begin{array}{c}M_{\mathrm{n}, \mathrm{M}}{ }^{a} \\
(\mathrm{~g} / \mathrm{mol})\end{array}$ & $\begin{array}{c}\text { PDI } \\
b\end{array}$ & $\begin{array}{c}f_{\mathrm{D}}{ }^{a} \\
(\%)\end{array}$ & $\begin{array}{c}f_{\mathrm{S}}{ }^{a} \\
(\%)\end{array}$ & $\begin{array}{c}f_{\mathrm{M}}{ }^{a} \\
(\%)\end{array}$ & $\begin{array}{c}T_{\mathrm{g}(\mathrm{PS})} \\
\left({ }^{\circ} \mathrm{C}\right)\end{array}$ & $\begin{array}{c}T_{\mathrm{g}(\mathrm{PMPCS})} \\
\left({ }^{\circ} \mathrm{C}\right)\end{array}$ \\
\hline $\begin{array}{c}\text { PDMS- } b \text {-PS- } b \text {-PMPCS } \\
\mathbf{D}_{\mathbf{5 8}} \mathbf{S}_{\mathbf{7 0}} \mathbf{M}_{\mathbf{1 0 0}}\end{array}$ & 4,300 & 7,300 & 40,400 & 1.07 & 11 & 16 & 73 & 102 & 109 \\
\hline
\end{tabular}


${ }^{a}$ Determined from ${ }^{1} \mathrm{H}$ NMR results of the block copolymers with the absolute molecular weight of PDMS macroinitiator $=4,300 \mathrm{~g} / \mathrm{mol}$. The volume fraction was calculated from the molecular weights and the mass density of PDMS $\left(0.97 \mathrm{~g} / \mathrm{cm}^{3}\right)$, PS $\left(1.05 \mathrm{~g} / \mathrm{cm}^{3}\right)$ and PMPCS $\left(1.28 \mathrm{~g} / \mathrm{cm}^{3}\right)$.

${ }^{b}$ Determined from GPC results using linear polystyrene standards.

${ }^{c}$ Determined from DSC results.

Table S2. Solubility parameters of polymers and solvents studied. ${ }^{7}$

\begin{tabular}{cc}
\hline Material & $\begin{array}{c}\text { Solubility } \\
\text { parameters }\left(\mathrm{MPa}^{1 / 2}\right)\end{array}$ \\
\hline Heptane & 15.2 \\
PDMS & 15.3 \\
PS & 18.5 \\
Acetone & 19.8 \\
PMPCS & 20.7
\end{tabular}

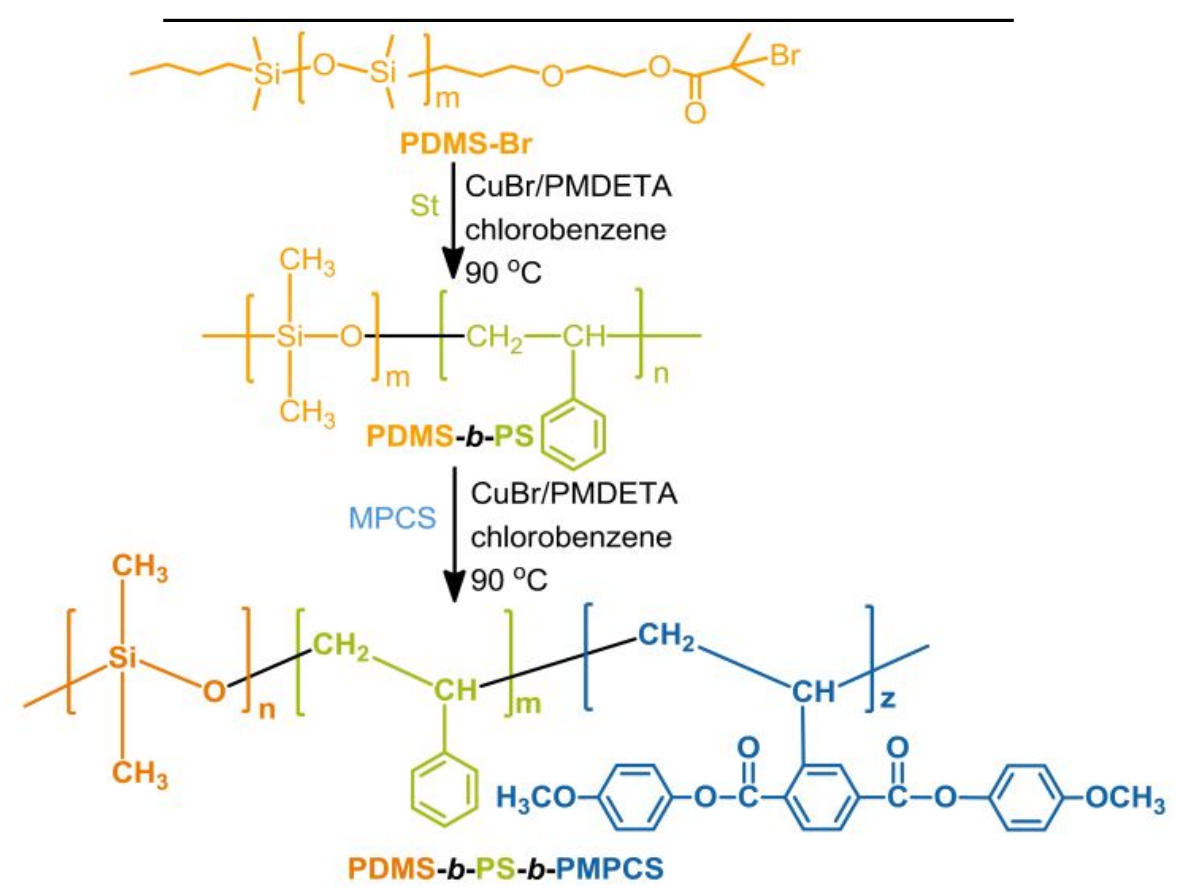

Scheme S1. Synthetic route of the PDMS- $b$-PS- $b$-PMPCS Triblock Terpolymer. 


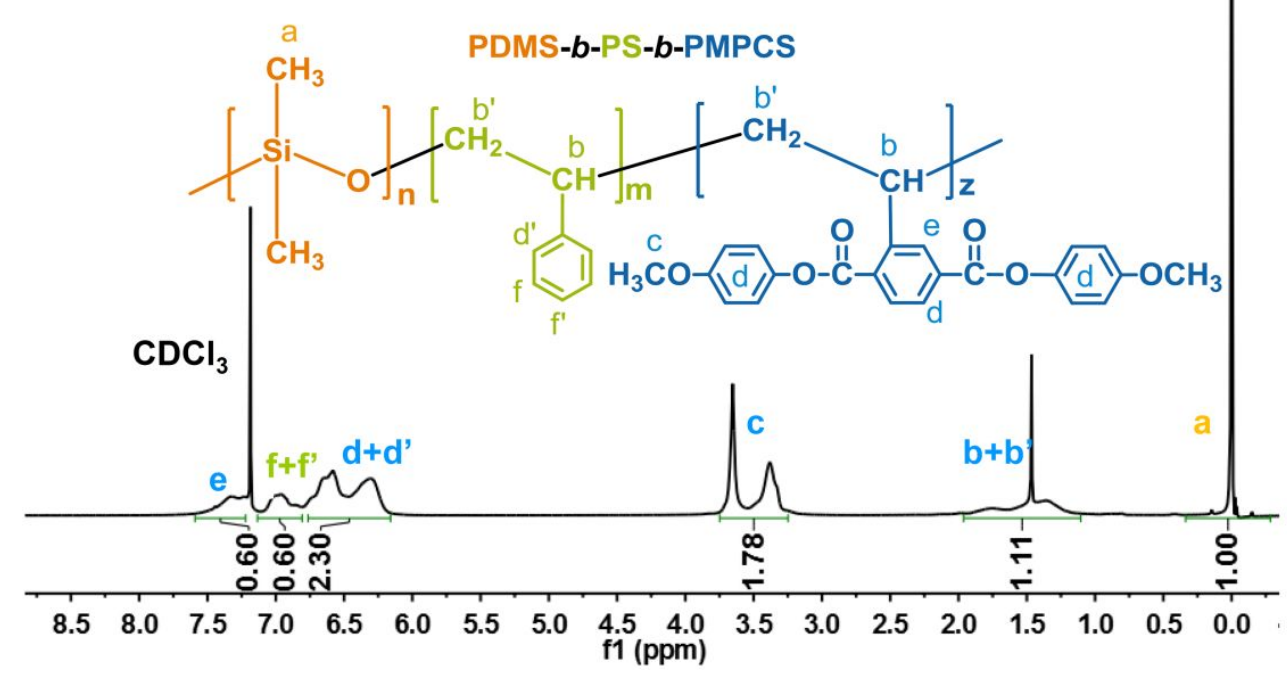

Figure S1. ${ }^{1} \mathrm{H}$ NMR spectrum of the PDMS- $b$-PS- $b$-PMPCS triblock terpolymer. The repeat units of the PS block was calculated to be 70 from the ratio of the integrated value of the resonance peak of the $\mathrm{H}$ $\left(\mathrm{f}+\mathrm{f}^{\prime}\right)$ on the phenyl group of the PS block to that of the $\mathrm{H}$ (a) on the $\mathrm{OCH}_{3}$ group of PDMS, and the repeat units of the PMPCS block was calculated to be 100 based on the ratio of the integrated value of the resonance peak of the $\mathrm{H}$ (c) on the $\mathrm{OCH}_{3}$ group of PMPCS to that of the $\mathrm{H}$ (a) on the $\mathrm{OCH}_{3}$ group of PDMS.

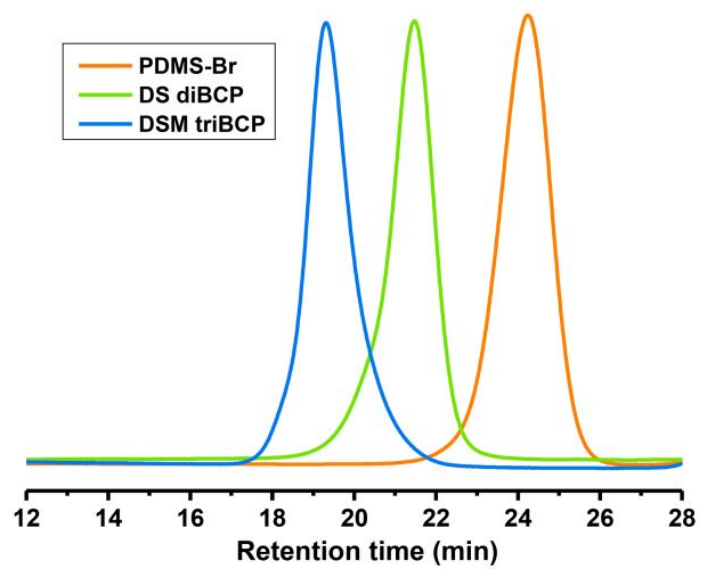

Figure S2. GPC curves of the PDMS macroinitiator, the PDMS- $b$-PS diblock copolymer, and the PDMS- $b$-PS- $b$-PMPCS triblock terpolymer after purification. 


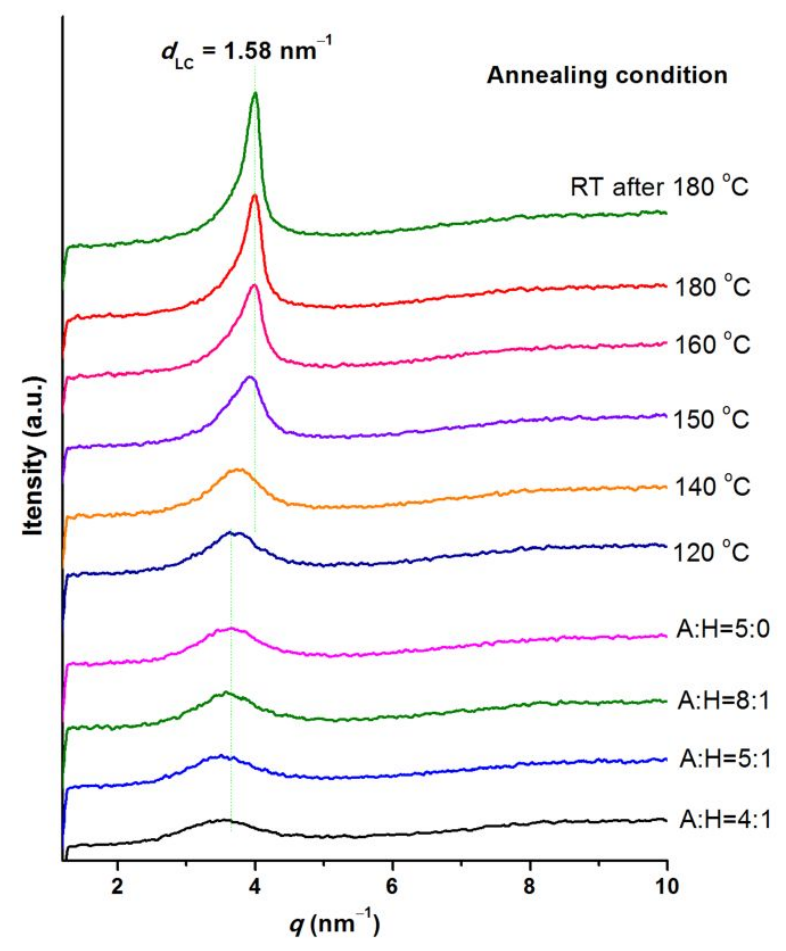

Figure S3. WAXS profiles of the PDMS- $b$-PS- $b$-PMPCS triblock terpolymer bulk films after solvent annealing and thermal annealing at indicated conditions.

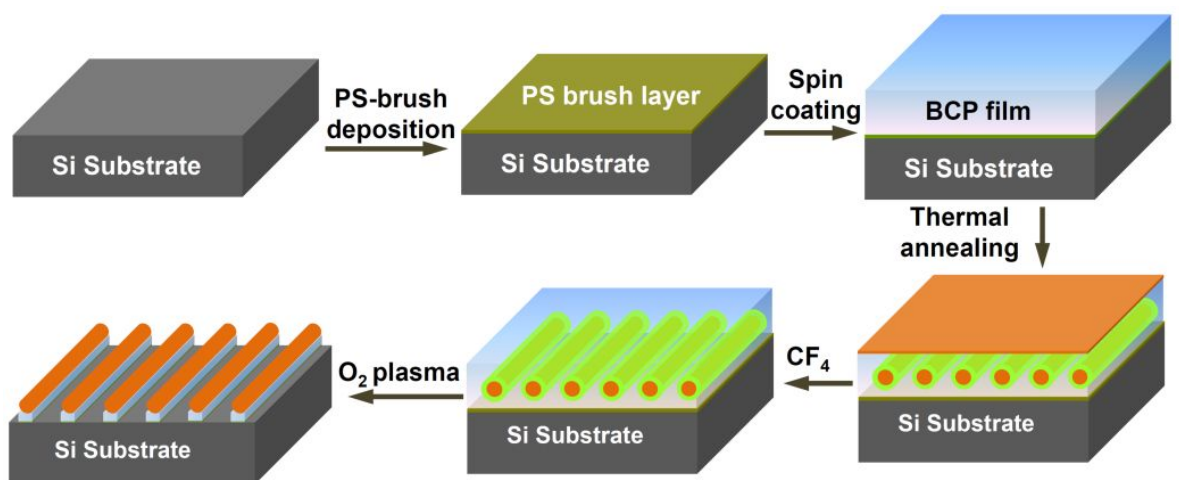

Figure S4. Schematics of DSM triblock terpolymer thin film self-assembly and subsequent plasma selective etching process. 

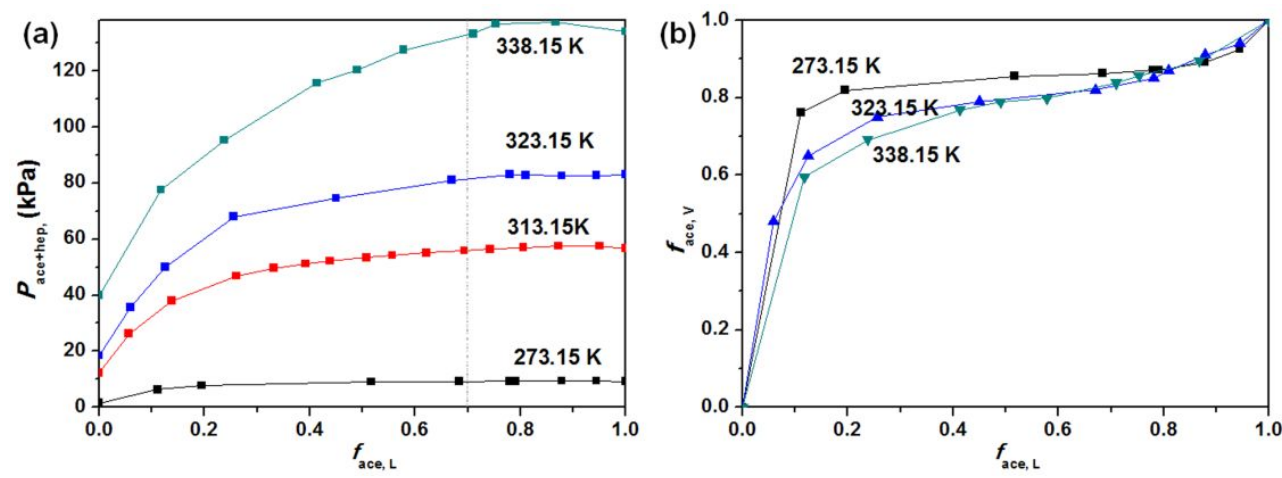

Figure S5. (a) Plots of total partial pressure of acetone and heptane $\left(P_{\text {ace+hep }}\right)$ as function of the mole fraction of the acetone in the liquid $\left(f_{\text {ace, }}\right)$. (b) Plots of mole fraction of the acetone in the vapor $\left(f_{\text {ace, }, \mathrm{V}}\right)$ as function of $f_{\text {ace, } \mathrm{L}}$ at different temperatures.

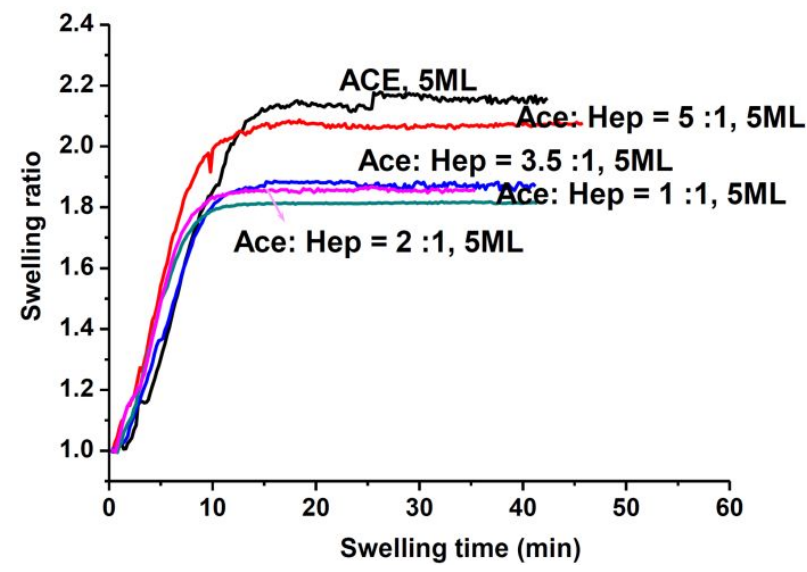

Figure S6. The profiles of swelling ratio as function of swelling time with different solvent mixtures. 


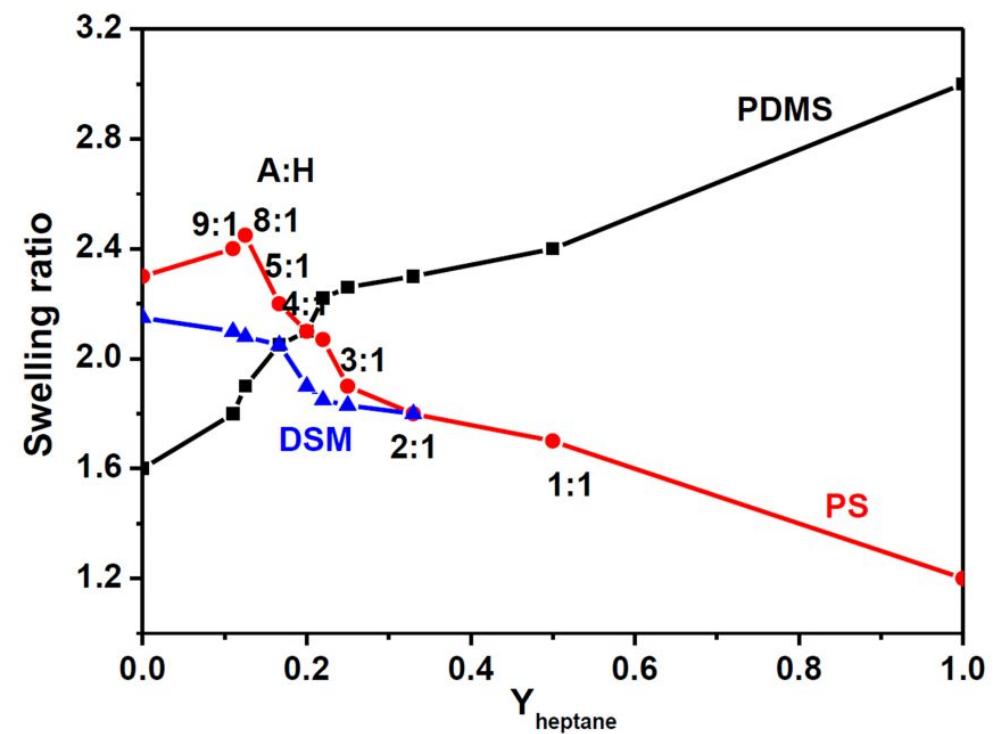

Figure S7. The plots of swelling ratios of PDMS, PS homopolymers, and DSM triblock terpolymer as a function of volume ratio of heptane in the mixed solvents used for SVA. 

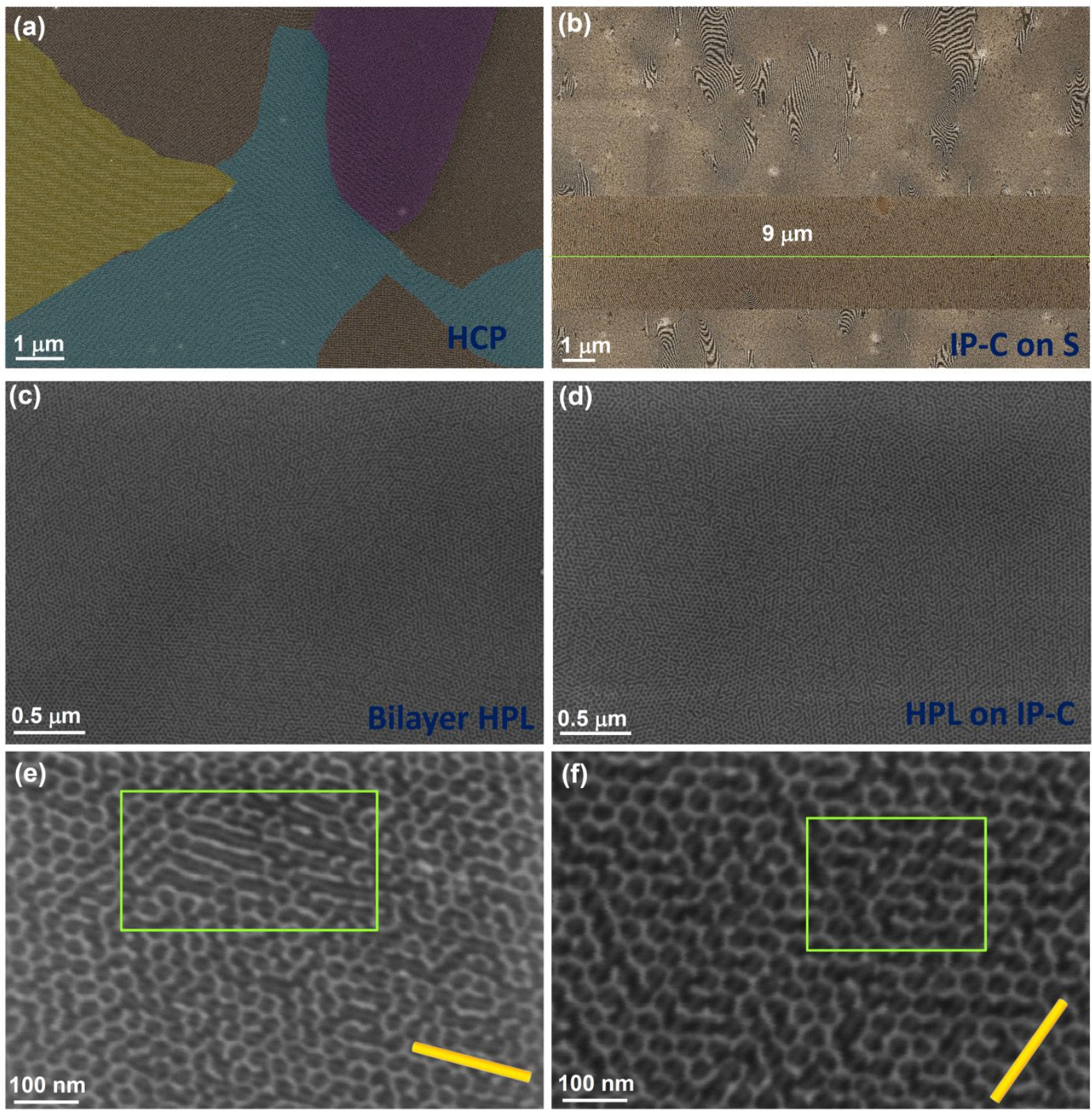

Figure S8. Representative low magnification SEM images revealing long-range ordered morphologies in DSM thin films after solvent vapor annealing with different acetone/heptane ratios. a) Double-layer hexagonally close packed (HCP) spheres formed in $30 \mathrm{~nm}$ initial thick film after annealing in pure acetone. b) Double-layer structure composed of spheres (S) in the bottom layer and in-plane cylinders (IP-C) in the top layer (IP-C on S) formed in $30 \mathrm{~nm}$ initial thick film after annealing with an acetone:heptane ratio of 5:1, with the inset presenting part of a single region of uniform orientation at least $9 \mu$ m wide. c) Double-layer hexagonally coordinated perforated lamellar (HPL) structure formed in 
a $30 \mathrm{~nm}$ thick film after annealing with an acetone:heptane ratio of 3.5:1. d) HPL on IP-C multilevel structure formed in a $65 \mathrm{~nm}$ thick film after annealing with an acetone:heptane ratio of 3.5:1. The corresponding morphologies are indicated. e,f) SEM images of HPL on IP-C thin film with the orientation of in-plane cylinders in the in-plane direction as indicated.
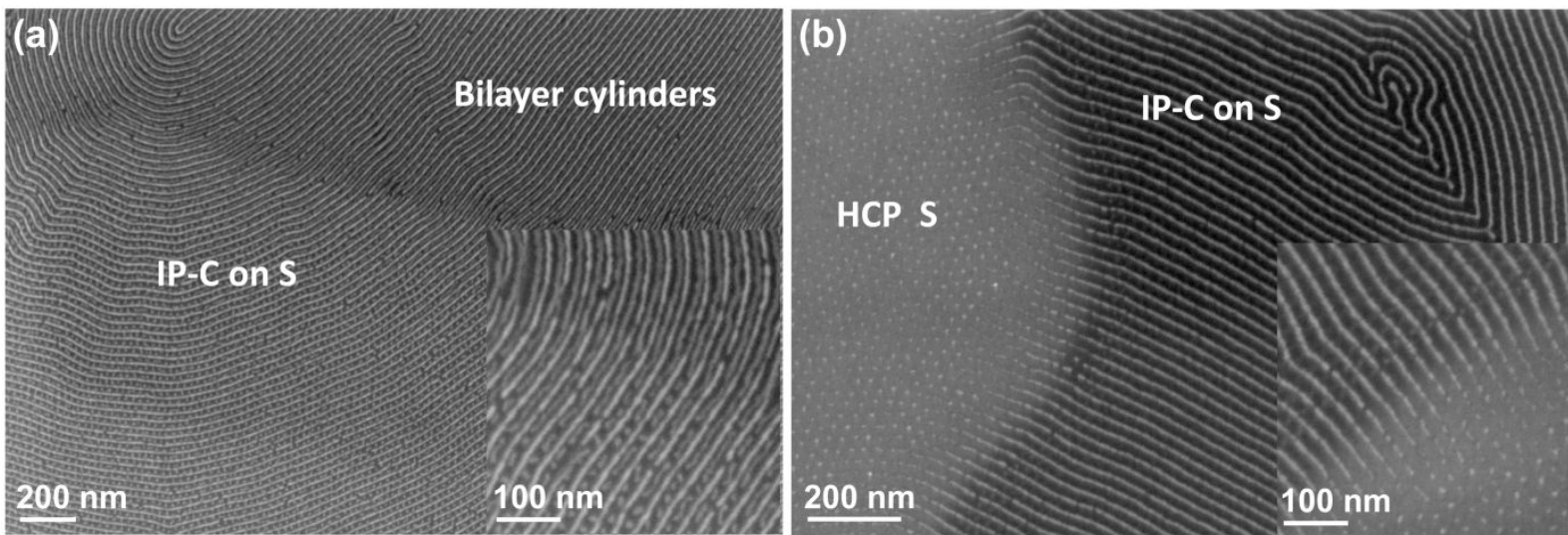

Figure S9. SEM images of oxidized PDMS patterns formed in the DSM thin films with initial thicknesses of $35 \mathrm{~nm}$ (left) and $20 \mathrm{~nm}$ (right) after annealing in the vapor from acetone:heptane 5:1 mixture. a) Terrace morphology composed of bilayer IP-C in the thicker regions and IP-C on S in thinner regions. b) Terrace morphology composed of IP-C on S in thicker regions and HCP spheres in thinner regions. 

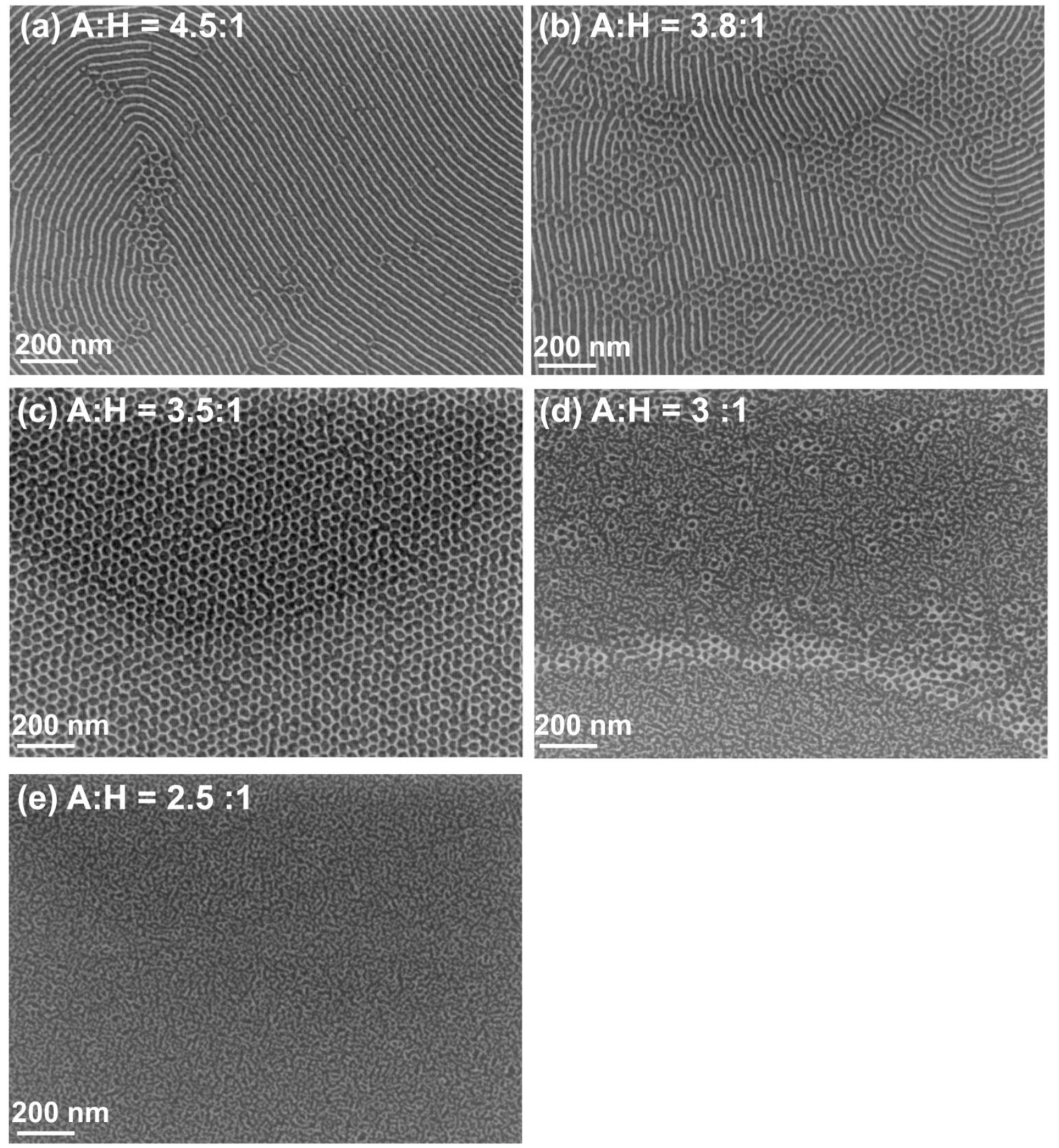

Figure S10. SEM images of oxidized PDMS patterns of the DSM thin film with initial thickness of 30 $\mathrm{nm}$ after annealing in vapor from acetone:heptane mixtures of $4.5: 1$ to $2.5: 1$. The morphology is transformed from mixed structure of HPL with cylinders $(a, b)$ to double-layer HPL $(c)$ and then to a disordered structure $(\mathrm{d}, \mathrm{e})$. 


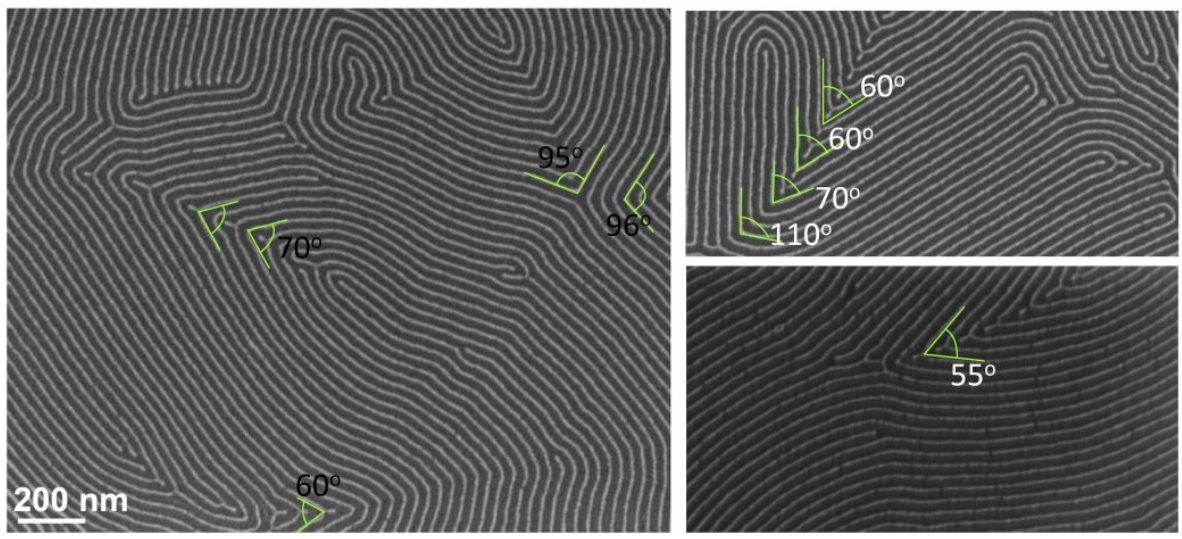

Figure S11. SEM images of cylindrical oxidized PDMS patterns with bends and arrow morphology in the DSM thin film with initial thickness of $30 \mathrm{~nm}$ after annealing in vapor from acetone:heptane mixtures of $7: 1$. The angles of the bends are labeled, from which it can be concluded that an arrow occurs when the bend angle is less than $70^{\circ}$.

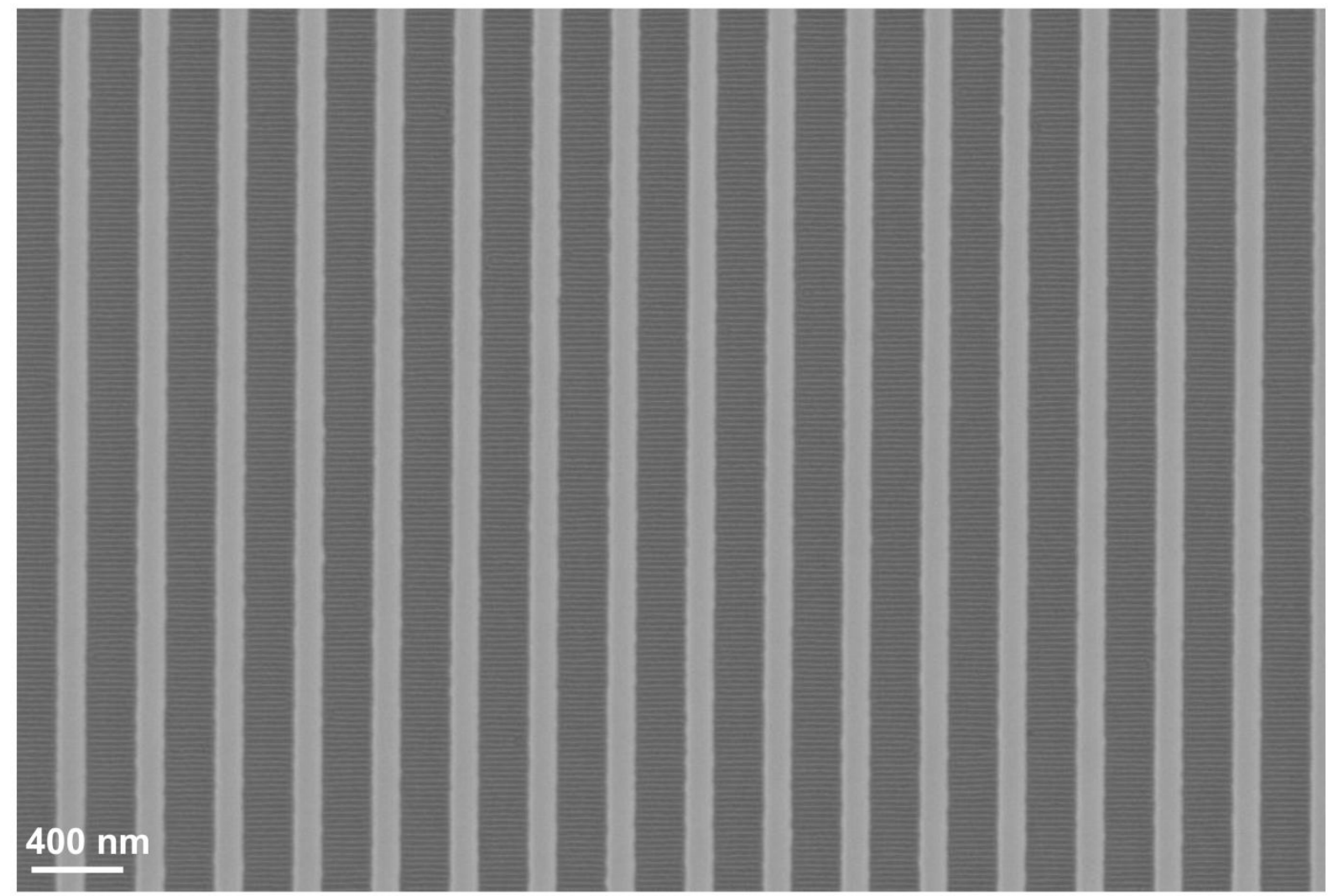

Figure S12. Low magnification SEM images of templated self-assembly of DSM terpolymer in $100 \mathrm{~nm}$ deep topographic trenches with trench width $230 \mathrm{~nm}$ after $30 \mathrm{~min}$ annealing in vapor from an 
acetone:heptane mixture of $7: 1$.

\section{References}

(1) Shi, L.-Y.; Shen, Z.; Fan, X.-H. Order-Order Transition in a Rod-Coil Diblock Copolymer Induced by Supercritical $\mathrm{CO}_{2}$. Macromolecules 2011, 44, 2900-2907.

(2) Gu, W.; Hong, S. W.; Russell, T. P. Orienting Block Copolymer Microdomains with Block Copolymer Brushes. ACS Nano 2012, 6, 10250-10257.

(3) Chen, L.-C.; Bai, W.; Fernandez, E.; Tu, K-H.; Ntetsikas, K.; Liontos, G.; Avgeropoulos, A.; Ross, C. A. Directed Self-assembly and Pattern Transfer from a High Molecular Weight Polystyrene-block-Poly(dimethylsiloxane) Block Copolymer Film. Nanotechnology 2017, 28, 145301.

(4) Bai, W.; Hannon, A. F.; Gotrik, K. W.; Choi, H. K.; Aissou, K.; Liontos, G.; Ntetsikas, K.; Alexander-Katz, A.; Avgeropoulos, A.; Ross, C. A. Thin Film Morphologies of Bulk-Gyroid Polystyrene-block-Polydimethylsiloxane under Solvent Vapor Annealing. Macromolecules 2014, 47,6000 .

(5) Wichterle, I.; Linek, J.; Wagner, Z.; Fontaine, J. C.; Sosnkowska-Kehiaian, K.; Kehiaian, H. V. In Binary Liquid Systems of Nonelectrolytes. Part 1; Kehiaian, H. V., Ed.; Springer Berlin Heidelberg: Berlin, Heidelberg, 2007, p 1.

(6) Maripuri, V. O.; Ratcliff, G. A. Journal of Chemical \& Engineering Data 1972, 17, 366.

(7) Barton, A. F. M. In Handbook of Polymer-Liquid Interaction Parameters and Solubility Parameters; CRC Press: Florida, 1990, p 125. 